\title{
Review Article \\ Genome Editing in Plants: An Overview of Tools and Applications
}

\author{
Venera S. Kamburova, ${ }^{1}$ Elena V. Nikitina, ${ }^{1}$ Shukhrat E. Shermatov, ${ }^{1}$ Zabardast T. Buriev, \\ Siva P. Kumpatla, ${ }^{2}$ Chandrakanth Emani, ${ }^{3}$ and Ibrokhim Y. Abdurakhmonov ${ }^{1}$ \\ ${ }^{1}$ Center of Genomics and Bioinformatics, Academy of Sciences of the Republic of Uzbekistan, University Street-2, \\ Qibray Region, 111215 Tashkent, Uzbekistan \\ ${ }^{2}$ Dow AgroSciences LLC, Indianapolis, IN 46268, USA \\ ${ }^{3}$ Western Kentucky University-Owensboro, Owensboro, KY 42303, USA
}

Correspondence should be addressed to Ibrokhim Y. Abdurakhmonov; genomics@uzsci.net

Received 20 April 2017; Accepted 28 May 2017; Published 3 July 2017

Academic Editor: Kent Burkey

Copyright (C) 2017 Venera S. Kamburova et al. This is an open access article distributed under the Creative Commons Attribution License, which permits unrestricted use, distribution, and reproduction in any medium, provided the original work is properly cited.

\begin{abstract}
The emergence of genome manipulation methods promises a real revolution in biotechnology and genetic engineering. Targeted editing of the genomes of living organisms not only permits investigations into the understanding of the fundamental basis of biological systems but also allows addressing a wide range of goals towards improving productivity and quality of crops. This includes the creation of plants with valuable compositional properties and with traits that confer resistance to various biotic and abiotic stresses. During the past few years, several novel genome editing systems have been developed; these include zinc finger nucleases (ZFNs), transcription activator-like effector nucleases (TALENs), and clustered regularly interspaced short palindromic repeats/Cas9 (CRISPR/Cas9). These exciting new methods, briefly reviewed herein, have proved themselves as effective and reliable tools for the genetic improvement of plants.
\end{abstract}

\section{Introduction}

Since the advent of recombinant DNA technology in Paul Berg's laboratory [1] in 1972, genetic engineering has come a long way and achieved enormous success. Many molecular and genetic mechanisms and phenomena have been discovered and studied in detail and the knowledge accumulated now permits researchers to reproduce experiments in vitro. Several decades-long investigations in molecular genetics and biochemistry of bacteria and viruses have allowed researchers to develop new methods of manipulating DNA through creation of various vector systems and tools for their delivery into the cell. All of these developments allow successful creation of not only transgenic microorganisms but also genetically modified higher organisms including various plant and crop species. Creation of novel tools for breeding and biotechnology, an application area of genetic engineering, has received significant focus resulting in accelerated development of useful tools. However, conventional genetic engineering strategy has several issues and limitations, one of which is the complexity associated with the manipulation of large genomes of higher plants [2].

Currently, several tools that help to solve the problems of precise genome editing of plants are at scientists' disposal. In 1996, for the first time, it was shown that protein domains such as "zinc fingers" coupled with FokI endonuclease domains act as site-specific nucleases (zinc finger nucleases (ZFNs)), which cleave the DNA in vitro in strictly defined regions [3]. Such a chimeric protein has a modular structure, because each of the "zinc finger" domains recognizes one triplet of nucleotides. This method became the basis for the editing of cultured cells, including model and nonmodel plants $[4,5]$.

Continued efforts and investigations led to the development of new genome editing tools such as TALENs (transcription activator-like effector nucleases) and CRISPR/Cas (clustered regularly interspaced short palindromic repeats). Designing TALENs requires reengineering of a new protein 
for each of the targets. However, the design process has been streamlined recently by making the modules of repeat combinations available that essentially reduces the cloning required for the design. On the other hand, designing and use of CRISPR are simple. Both TALEN and CRISPR systems have been shown to work in human cells, animals, and plants. Such editing systems when used for efficient manipulation of genomes could solve complex problems including the creation of mutant and transgenic plants $[12,41]$. Moreover, chimeric proteins containing zinc finger domains and activation domains of other proteins and those based on the TALE DNA-binding domain and Cas9 nuclease were used in experiments for regulation of gene transcription, study of epigenomes, and the behavior of chromosome loci in cell cycle [24, 42-44].

In this review, we briefly described the mechanisms of different genome editing systems and their use for crop improvement and also highlighted the multiple advantages and applications of engineered nucleases as well as biosafety and regulatory aspects of plants generated using engineered nuclease based technologies.

\section{Mechanisms of Genome Editing Systems}

Novel genome editing tools, also referred to as genome editing with engineered nuclease (GEEN) technologies, allow cleavage and rejoining of DNA molecules in specified sites to successfully modify the hereditary material of cells. To this end, special enzymes such as restriction endonucleases and ligase can be used for cleaving and rejoining of DNA molecules in small genomes like bacterial and viral genomes. However, using restriction endonucleases and ligases, it is extremely difficult to manipulate large and complex genomes of higher organisms, including plant genomes. The problem is that the restriction endonucleases can only "target" relatively short DNA sequences. While such specificity is enough for short DNA viruses and bacteria, it is not sufficient to work with large plant genomes. The first efforts to create methods for the editing of complex genomes were associated with the designing of "artificial enzymes" as oligonucleotides (short nucleotide sequences) that could selectively bind to specific sequences in the structure of the target DNA and have chemical groups capable of cleaving DNA [45].

Targeted approach to address this challenge was the design of chimeric nucleases which are complex proteins containing one or two structural units, one of which catalyzes the cleavage of DNA, and the second is capable of selectively binding to specific nucleotide sequences of target molecule, providing the nuclease action to this site (Table 1) [46, 47]. These chimeric nucleases can be "produced" directly in the cell: to this end, appropriately engineered vectors encoding nucleases need to be introduced into cell. Such vectors are also supplied with nuclear localization signal which enables the nuclease to enter the cell nucleus thereby getting access to genomic DNA.

2.1. Zinc Finger Nucleases (ZFNs). ZFNs were the first generation of genome editing tools that use chimerically engineered nucleases which were developed after the discovery of the working principles of the functional Cys2-His2 zinc finger (ZF) domain [3, 4, 46, 48]. Each Cys2-His2 ZF domain consists of 30 amino acid residues, which are folded up to $\beta \beta \alpha$ configuration [48-50]. Crystallographic structure analysis showed that the Cys2-His2 ZF proteins bind to DNA by inserting an $\alpha$-helix of the protein into the major groove of the DNA-double helix [51]. Each ZF protein has the ability to recognize 3 tandem nucleotides in the DNA. Generalized ZFN monomer consists of two different functional domains: artificial ZF Cys2-His2 domain at the $\mathrm{N}$-terminal region and a nonspecific FokI DNA cleavage domain at the Cterminal region. FokI domain dimerization is critical for $\mathrm{ZFN}$ enzymatic activity [3]. The observation that the modular recognition of zinc finger domains presents as a series to the corresponding, consecutive three bp targets enabled the realization that each of the individual zinc finger domains could be interchangeable and that the manipulation of the order of the domains would lead to unique binding specificities to the proteins harboring them thereby enabling targeting of specific, unique sequences in the genome. For example, a ZFN dimer, consisting of two 3 or $4 \mathrm{ZF}$ domains, recognizes a target sequence of 18 or 24 base pairs, which statistically form unique sites in the genomes of most organisms (Table 1).

The design and application of ZFNs involve modular design, assembly, and optimization of zinc fingers against specific target DNA sequences followed by linking of individual ZFs towards targeting larger sequences. Over the years, zinc finger domains have been generated to recognize a large number of triplet nucleotides. This enabled the selection and linking of zinc fingers in a sequence that would permit recognition of the target sequence of interest.

Since the first report on zinc fingers in 1996, they have been successfully used in several organisms including plants [4]. Examples include targeted inactivation of endogenous genes in Arabidopsis [15, 16], high frequency modification of tobacco genes [17], and precise targeted addition of a herbicide-tolerance gene as well as insertional disruption of a target locus in maize [18]. ZFNs have also been used for trait stacking in maize $[52,53]$.

Zinc finger nucleases have revolutionized the field of genome editing by demonstrating the ability to manipulate genomic sites of interest and opened the gates for both basic and applied research. ZFNs provide advantages over other tools with respect to efficiency, high specificity, and minimal nontarget effects and current efforts are focused on further improving design and delivery as well as expanding their applications in diverse crops of interest.

\subsection{Transcription Activator-Like Effector Nucleases (TALENs).} The quest for efficient and selective manipulation of target genomic DNA led to the identification of unique transcription activator-like effector (TALE) proteins that recognize and activate specific plant promoters through a set of tandem repeats that formed the basis for the creation of a new genome editing system consisting of chimeric nucleases called TALE nucleases (TALENs) [47]. TALE proteins consist of a central domain responsible for DNA binding, nuclear localization 
|⿹弋龴

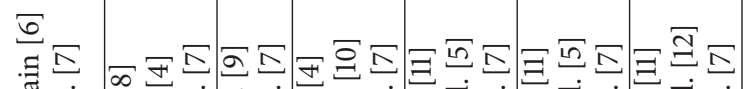

竎

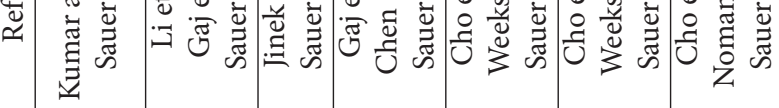

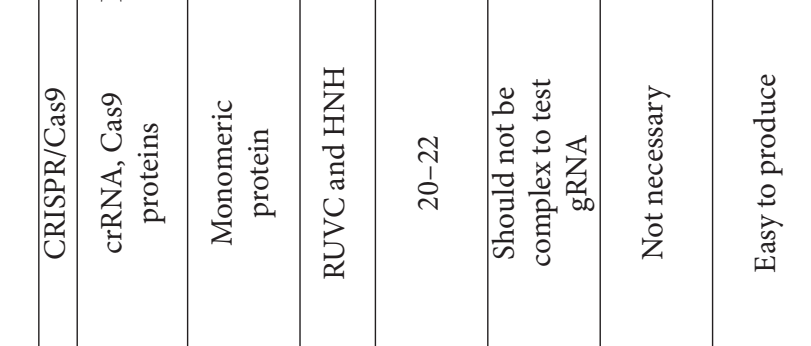

0
0
0
0
0
0
0
0
0
0
0
0
0
0
0
0
0
0
0
0
0
0
0

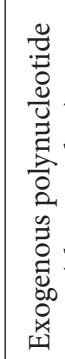

๖ 灵 


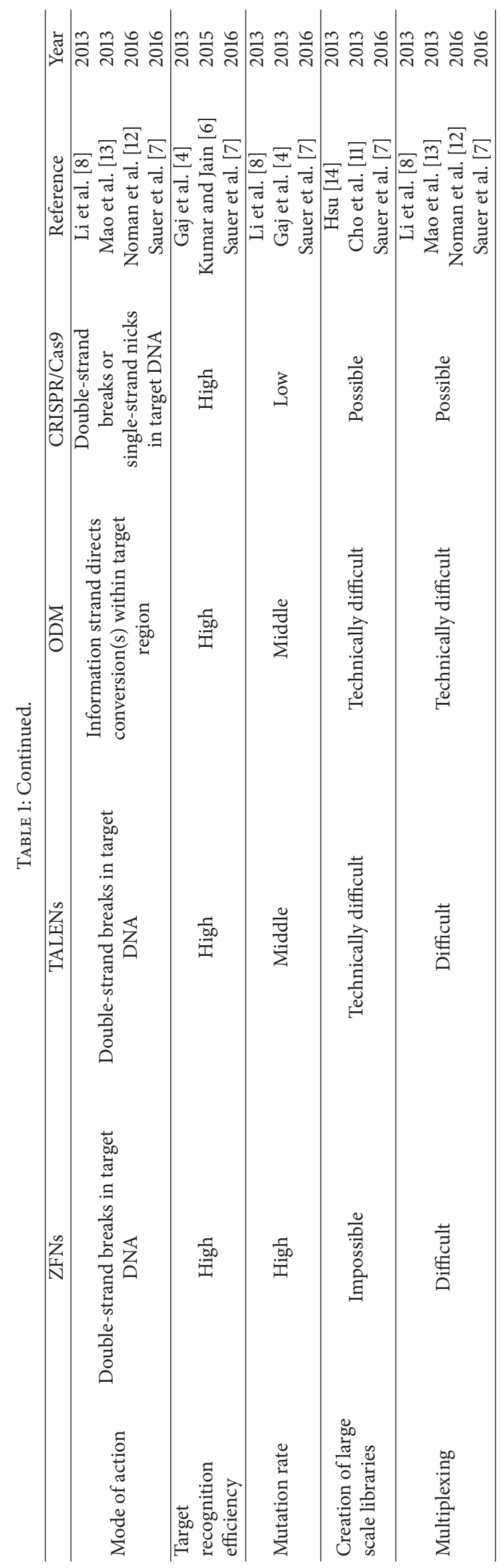


signal, and a domain that serves as activator of transcription of the target gene (Table 1) [54]. For the first time, the DNAbinding ability of these proteins was described in 2007 [55], and a year later, two scientific groups have decoded the recognition code of target DNA sequence by TALE proteins [56].

It is shown that the DNA-binding domain in TALE monomers in turn consists of a central repeat domain (CRD) that confers DNA binding and host specificity. The CRD consists of tandem repeats of 34 amino acid residues and each 34-amino acid long repeat in the CRD binds to one nucleotide in the target nucleotide sequence. Two of the amino acids of the repeat, located at positions 12 and 13, are highly variable (repeat variable diresidue (RVD)) and are responsible for the recognition of specific nucleotide with degeneracy of binding several nucleotides with differential efficiency. The last tandem repeat binding to nucleotide at the $3^{\prime}$-end of the recognition site consists of 20 amino acid residues only and, therefore, it is named as half-repeat. While TALE proteins, in general, can be designed to bind any DNA sequence of interest, studies have demonstrated that the $5^{\prime}$-most nucleotide base of the DNA sequence bound by a TALE protein should always be a Thymidine and that a deviation from this requirement can affect the efficacy of TALE transcription factors (TALE-TF), TALE recombinases (TALE-R), and TALENs [57].

After the DNA code recognition requirements by TALE proteins have been cracked, the very first effort undertaken was the creation of chimeric TALEN nucleases [5]. For this purpose, the sequence encoding the DNA-binding TALE domain was inserted into a plasmid vector previously used to create ZFN [58]. This resulted in the creation of a synthetic, chimeric sequence-specific nuclease genetic construct containing the DNA-binding domain of TALEs and the catalytic domain of FokI restriction endonuclease. This construct helped to create artificial nucleases with DNAbinding domain and different RVDs that can target any nucleotide sequence of interest $[2,4]$.

In most studies, the monomers with RVDs Asn and Ile (NI), Asn and Gly (NG), two Asn (NN), and His and Asp (HD) bind to nucleotides A, T, G, and C, respectively. NN, the most common RVD that specifies G, was also found to bind to A. This suboptimal or lack of specificity is a concern for the use of engineered TALEs for targeting DNA. Another RVD NK has less functional efficiency compared to $\mathrm{NN}$, although it has demonstrated guanine specificity. Several studies have also shown that the use of NH or NK RVDs for specific binding of guanine reduces the risk of nontarget effects $[19,59,60]$. It has been shown that in RVD (NI, NG, $\mathrm{NN}$, or $\mathrm{HD}$ ) the first amino acid residue, whether it is $\mathrm{N}$ or $\mathrm{H}$, is responsible for the stabilization of spatial conformation although it does not directly bind to a nucleotide, whereas the second amino acid residue binds to a nucleotide either through hydrogen bonding with nitrogenous bases (in case of $\mathrm{D}$ and $\mathrm{N}$ amino acids) or through van der Waals forces (in case of I and G) [61].

Based on the mode of action and specificity of TALENs, it should be possible to introduce double strand breaks in any location of the genome as long as that location harbors the recognition sequence corresponding to the DNA-binding domains of TALENs. There is another condition that also needs to be met, that is, the requirement of the presence of Thymidine before the $5^{\prime}$ end of the intended target sequence since it has been demonstrated that the W232 residue in the $\mathrm{N}$-terminal portion of the DNA-binding domain interacts with the Thymidine and influences the binding efficiency [62]. It is also possible to overcome this $5^{\prime}$ Thymidine constraint by developing mutant variants of TALEN Nterminal domain which can bind other nucleotides [57]. Considering the ease of site-directed manipulation using TALEN system, within a short period of time after the unraveling of the TALEN mode of action, the genes modified by this system have been used successfully in several animal and plant species and the plant examples include rice, wheat, Arabidopsis, potato, and tomato (Table 2) [63].

\subsection{Oligonucleotide-Directed Mutagenesis (ODM). After first} successful exploitation in mammalian systems, oligonucleotide-directed mutagenesis (ODM) has become another novel gene editing tool for plants [7, 64]. ODM, a tool for targeted mutagenesis, uses a specific 20- to 100-base long oligonucleotide, the sequence of which is identical to the target sequence in the genome except that it contains a single base pair change (intended mutation to be inserted in the genome) towards achieving site-directed editing of gene/sequence of interest (Table 1) [65]. When these synthetic oligonucleotides or repair templates with homology to a specific region of the target gene are transiently exposed to the plant cells by using a variety of specific delivery methods, they bind to the targets and activate cell's natural repair machinery which recognizes the single mismatch in the template and then copies that mismatch or mutation into the target sequence through repair process $[7,65]$. This produces the desired targeted single nucleotide or base editing in the plant genome that confers novel function or trait while the plant cell degrades the repair template oligonucleotide. Using tissue culture methods, cells with edited sequences are subsequently regenerated and genome edited novel varieties with improved traits/characteristics are developed through traditional breeding (Table 2) [7, 64, 65].

\subsection{Clustered Regularly Interspaced Short Palindromic Repeats} (CRISPR). Another novel genome editing system that has emerged recently and has become widely popular is the clustered regularly interspaced short palindromic repeats (CRISPR)/CRISPR associated (Cas) protein system with the most prominent being the CRISPR/Cas9 (based on Cas9 protein). This is a method that utilizes adaptive bacterial and archaeal immune system, the mechanism of which relies on the presence of special sites in the bacterial genome called CRISPR loci. These loci are composed of operons encoding the Cas9 protein and a repeated array of repeat spacer sequences. The spacers in the repeat array are short fragments that are derived from foreign DNA (viral or plasmid) that have become integrated into bacterial genome following recombination $[41,66]$. 


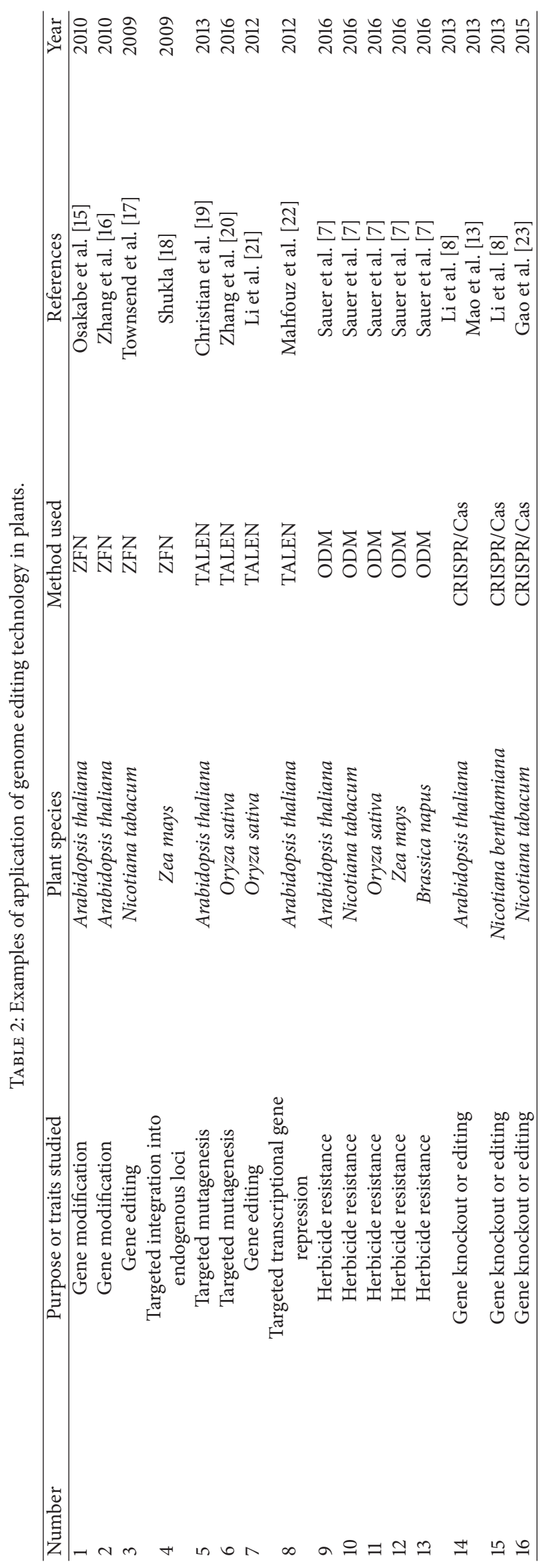




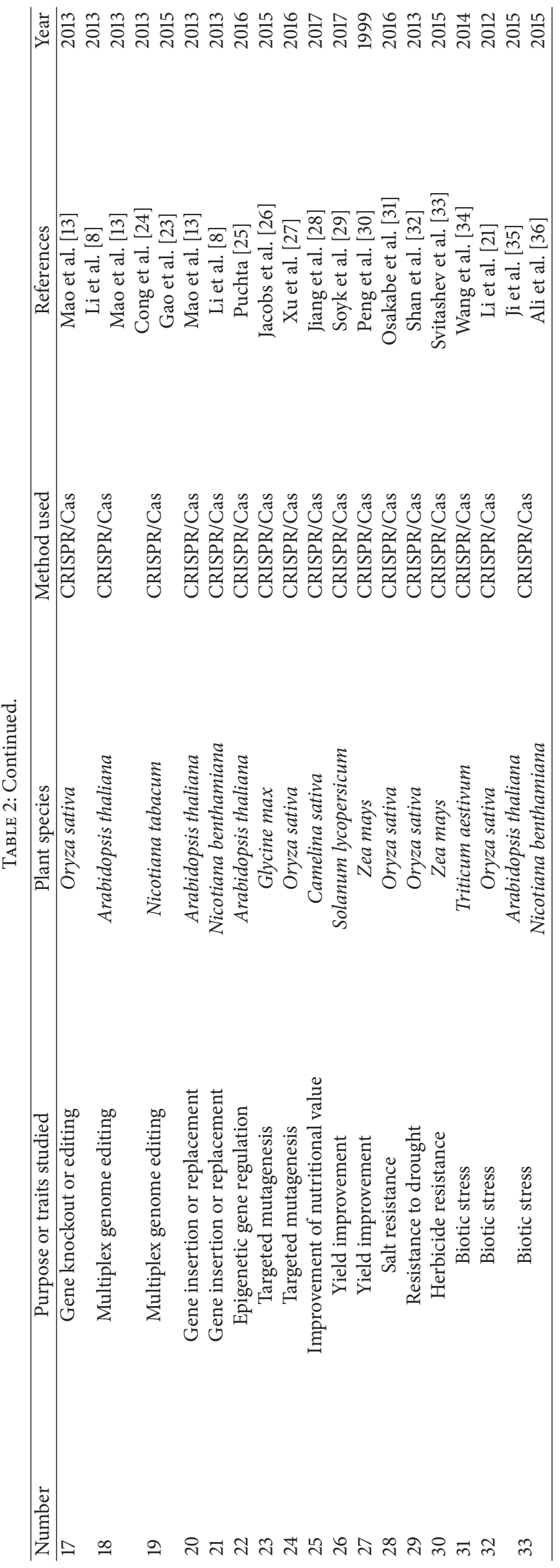




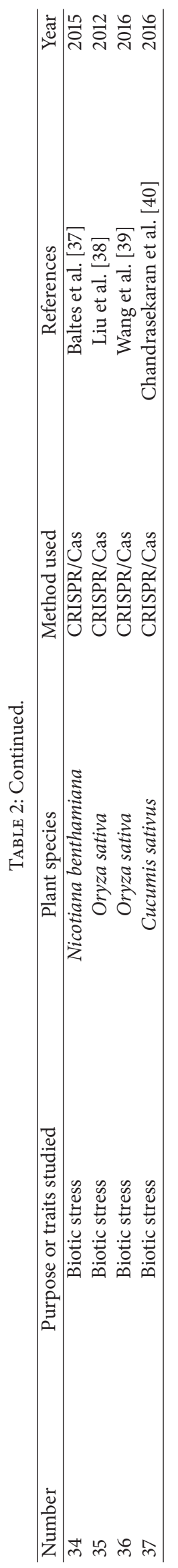


Unlike the chimeric TALEN proteins, target site recognition by CRISPR/Cas9 system is accomplished by the complementary sequence based interaction between the guide (noncoding) RNA and DNA of the target site and the guide RNA and Cas protein complex has the nuclease activity for exact cleavage of double-stranded DNA using Cas9 endonuclease (Table 1) $[9,24,67]$.

Several types of CRISPR protective systems functioning in cells of various bacteria are described in detail elsewhere $[68,69]$. The most "popular" system is the CRISPR/Cas type II-A system found in the bacterium Streptococcus pyogenes and composed of three genes encoding CRISPR RNA (crRNA), trans-activating crRNA (tracrRNA), and Cas9 protein. Based on this system, universal genetic constructs encoding artificial elements of CRISPR/Cas "genome editor" have been created [70]. Also, a simplified version of the system, functioning as a complex of Cas9 protein and a single guide RNA, consisting of CRISPR tracrRNA and short, mature crRNA was created. The guide sequence identifies the target DNA site and binds to it based on complementarity and Cas9 cleaves the DNA in target point [71].

CRISPR system can be used for the creation of genetically modified cells grown in culture and living organisms [11]. In the first case, plasmids or viral vectors which provide high and stable synthesis of CRISPR/Cas9 system elements are introduced into cells. In the second case, cultured protoplasts and a plasmid coding CRISPR/Cas elements are used to obtain genetically modified plants [32]. Another approach, applied for plants, is the use of Agrobacterium, the natural "genetic engineer," that contains a special plasmid harboring CRISPR/Cas9 system [41, 44].

Thus, due to its simplicity, efficiency, and wide capabilities, in a short time CRISPR/Cas9 system has already found use in various fields of fundamental and applied biology, biotechnology, and genetic engineering.

2.5. Repair of Cleaved Genomic Sites. An important step in the genome editing process is the repair of the DNA break created by the nucleases. DNA break gets repaired by the endogenous cellular mechanisms: nonhomologous endjoining (NHEJ) or homology-dependent (or directed) repair (HDR) [14]. NHEJ is the simplest mechanism where the ends of the cleaved DNA are joined together, often resulting in the insertion or deletion of nucleotides (indels) thereby shifting the gene reading frame, resulting in a gene "knockout" [72]. If indels are not observed, the DNA is recovered, and there are no noticeable changes. On the other hand, HDR is a mechanism where a sequence containing homology to target is used as a template for repairing the break or the DNA lesion. Therefore, by providing a template that contains a desired sequence of interest flanked by sequences homologous to both sides of the break point, one can force the insertion of that desired sequence into the target site. When HDR occurs, a homologous recombination is used to enable new sequences for gene recovery or insertion [72]. This method is simple, provides the exact impact on DNA target, and can be used at almost any modern molecular biology laboratory.

\section{Practical Applications of Genome Editing Systems}

3.1. Application of "Genome Editors" for Functional Genomics. Several different types of genome modifications can be achieved by utilizing ZFN, TALEN, ODM, and CRISPR/Cas genome editing systems (Table 2). These include creation of point mutations, insertion of new genes in specific locations or deletion of large regions of the nucleotide sequences, and correction or substitution of individual genetic elements and gene fragments $[4,6,10,20,23,44,73]$.

While introducing modifications to various genomic elements in plant cells and examining the results, scientists were able to investigate the role of individual genes in the functioning of individual cells and the organism as a whole. For example, the unique ability of CRISPR/Cas9 system to selectively bind to specific DNA sites has helped to regulate gene activity $[24,41,44]$. For this purpose, proteins activating or repressing the activity of promoters that control the gene function can be attached to the catalytically inactive mutant Cas9 protein. In one example, it was shown that complex binding to the target DNA can inhibit or stimulate the function of the target gene [44].

Furthermore, using CRISPR/Cas9 system, several genetic constructs targeted to different genome sites can simultaneously be introduced into cells $[8,24,43]$. This is a welcome feature in investigating intergenic interaction, if any, because several genes are simultaneously affected by the CRISPR/Cas9 system [44]. For example, using this approach, it was possible to identify genes involved in crop domestication process [74].

\subsection{Application of Genome Editing Systems in Crop Improve-} ment. Genome editing technologies have wide practical applications for solving one of the most important tasks of modern biotechnology - the creation of new varieties of crops, which are high-yielding and resistant to abiotic and biotic stresses and also have high nutritional value (Table 2) $[31,63,75-80]$. To this end, genome editing system has been used in plant breeding (1) to insert point mutations similar to natural SNPs $[26,27],(2)$ to make small modifications to gene function [13], (3) for integration of foreign genes, (4) for gene pyramiding and knockout, and (5) for the repression or activation of gene expression, as well as (6) epigenetic editing [6].

For example, the use of ZFN in Arabidopsis thaliana [1517] and Zea mays [18] has led to the successful development of herbicide tolerant genotypes through insertion of herbicideresistance genes into targeted sites in the genome [18]. ZFN was also used for the targeted modification of an endogenous malate dehydrogenase $(\mathrm{MDH})$ gene in plants and the plants containing modified MDH have shown increased yield [81]. ODM technique has been significantly advanced through Cibus Rapid Trait Development System (RTDS) [7] and this technology has been successfully applied in several crops. Applications include but are not limited to the generation of herbicide tolerance, insect resistance, enhanced disease resistance (bacterial and viral), improved nutritional value, and enhanced yield without the introduction of foreign genes as has been used in traditional genetic engineering approach 
for crop development $[7,65]$. A precise editing of CAC to TAC using ODM RTDS technology has been demonstrated that converts BEP to GFP by changing Histidine (H66) to Tyrosine (Y66) in GFP protein. This approach has offered a nontransgenic breeding tool for crops $[7,64]$.

Using the CRISPR/Cas9 technology, Jiang et al. [28] have obtained "a biotech" oil from Camelina sativa seeds with an improved fatty acid composition, which makes it more beneficial to human health, more resistant to oxidation, and more appropriate for the production of certain commercial chemicals including biofuels [28]. Soyk et al. [29] used targeted mutagenesis of SP5G gene of tomato to create plants with rapid flowering and more compact bush, which in turn resulted in earlier harvest. In another effort, Osakabe et al. [31], using the CRISPR-induced mutagenesis of OST2 gene in Arabidopsis, were able to obtain new alleles that confer salt stress resistance to plants [31].

Modulation of the gibberellin biosynthesis by genome editing methods has allowed creation of dwarf fruit trees [30], which have great potential for increasing productivity through higher density plantings and reduced labor costs. This results in a reduction of land, water, pesticide, and fertilizer use [82]. In addition, genome editing for inhibition of ethylene biosynthesis, which plays a very important role in fruit ripening process [82] or its signaling pathways, enables creation of new varieties with extended shelf life [63].

A major area of application of genome editing approaches in plant breeding is to create varieties resistant to various pathogens and/or pests. These methods have been used for the modification of the key plant immunity stages at different levels in several crops. This goal can be achieved by modifying (1) susceptibility genes (S-genes), (2) resistance genes (R-genes), (3) genes regulating the interaction between the effector and target, and (4) the genes regulating plant hormonal balance [78]. For example, wheat genotypes resistant to powdery mildew disease were obtained by TALENand CRISPR/Cas9-mediated genome editing on mildewresistance locus $\mathrm{O}$ (MLO) [34]. Genome editing technologies have also been used to produce plants resistant to bacterial leaf blight, caused by Xanthomonas oryzae pv. oryzae [21].

The CRISPR/Cas9 system has been investigated for its efficacy in providing interference against geminiviruses by using a transient transformation system such that $N$. benthamiana degradation/suppression of curly top virus genome by single guide RNA/Cas9 (sgRNA/Cas9) has been demonstrated [35]. In other efforts, where sgRNAs specific for tomato yellow leaf curl virus (TYLCV) or bean yellow dwarf virus (BeYDV) sequences were introduced into $N$. benthamiana plants expressing Cas9 endonuclease and challenged with the corresponding viruses, it was demonstrated that the CRISPR/Cas9 system not only targeted viruses for degradation but also introduced mutations at the target sequences $[36,37]$ due to interference with the copy number of freely replicating viruses [78].

Metabolic pathways that regulate hormonal balance can also be modified using the genome editing technologies to enhance the immunomodulatory component of the plants immune system. This can be achieved by deactivating the ethylene-responsive factor (ERF). In particular, ethylenedependent pathway in rice has been successfully modified by CRISPR/Cas9-mediated target OsERF922 gene mutations, resulting in increased resistance to Magnaporthe oryzae [38, 39].

CRISPR/Cas9 has been used to knock out eIF4E gene that encodes the eukaryotic translation initiation factor essential for translation of viruses, in Cucumis sativus, and that knockout confers resistance to viruses such as cucumber vein yellowing virus (CVYV), zucchini yellow mosaic virus (ZYMV), and papaya ring spot mosaic virus-W (PRSV-W) [83]. In addition, CRISPR/Cas9 was demonstrated to be an efficient system for rapid and efficient genome editing in Phytophthora sojae, an oomycete pathogen of Soybean, by modifying the pathogenicity gene (Avr4/6), thereby opening up an avenue for the much needed functional genomics work in Phytophthora sojae towards the ultimate goal of controlling this pathogen [83].

Similarly, existing genome editing methods, in particular, CRISPR/Cas9 method, have been successfully used to obtain plants resistant to herbicides [33]. For example, editing of ALS2 gene in maize (acetolactate synthase or ALS is a key enzyme in the biosynthesis of amino acids in plants and has been inhibited by sulfonylurea herbicides) allowed the creation of a mutant corn plant resistant to chlorsulfuron [33].

Another interesting area of biotechnology where CRISPR/Cas9 system has significant application is the development of plants capable of synthesizing human proteins such as insulin, necessary for patients with diabetes mellitus, or albumin, which is used in the treatment of hemorrhagic shock, burns, hypoproteinemia, and cirrhosis [84]. At present, albumin is prepared from human plasma which is in a very limited supply; however, global demand for albumin is constantly growing and currently is equal to 500 tons per year. To meet the growing needs human albumin gene is already introduced into rice genome using genomic engineering techniques [85]. Such expressed proteins can be isolated from plant and animal tissues, where it is synthesized, and after clarification, it can be used for medical purposes.

Thus, as described above and extensively referenced herein, these novel genome editing techniques are being widely used for the purpose of crop improvement including new bioenergy crop developments [86]. However, the use of tissue culture with these GEEN methods may also create complexities that could slow the process of genome editing.

\section{Safety Assessment Aspects of Genome Editing Systems}

4.1. Nontarget Effects. Genome editing techniques, in essence, preserve the native genomic structure and, therefore, are considered as a safe technology for crop improvement. Despite this general understanding, there are some concerns related to the biosafety of crops created using these methods. One main concern in terms of its biosafety is the possibility of nontarget effects of synthetic nucleases during genome editing.

During the biotechnological application of genome editing methods, efficiency and specificity of the engineered 
nucleases are the two most important functional requirements and are closely related to the choice of the target site. For each endogenous genomic locus, efficiency of DNA cleavage (both target and nontarget) depends not only on the nuclease activity (such as FokI domains and Ruv domains of the Cas 9 proteins), but also on the availability of a target site and affinity of the DNA-binding domain (e.g., TAL effector domains and guide RNA, gRNA) to the target sequence. Specificity of engineered nucleases largely depends on the binding affinity of nuclease-DNA, including the binding of zinc finger to DNA (ZFNs), TAL effector to DNA (TALENs), and hybridization of gRNA with DNA (CRISPR), although dimerization of FokI domain (ZFNs and TALENs) and Cas9 interaction with the motif contiguous to protospacer adjacent motif (PAM) may also play an important role [87]. In case of ZFNs, while examples abound with respect to the binding efficiency of canonical $\mathrm{C} 2 \mathrm{H} 2$ binding domain containing ZFNs, investigations on the utility of noncanonical ZFNs such as those containing $\mathrm{C} 3 \mathrm{H} 1$ binding domain have demonstrated high levels of binding efficiency [88].

To minimize nontarget effects of genome editing systems, a crucial aspect is the careful selection of sites for the introduction of the double-stranded breaks by performing a prior bioinformatics analysis [89]. When choosing the desired sites, sites of repeated sequences and sites having a high homology with other regions of the genome should be avoided. In this regard, to facilitate the selection of the target sites for nucleases and experimental verification of the presence of nontarget effects, several software packages were developed that enable nuclease design and validation $[79,87,90]$.

4.2. Regulation of Plants Created by Genome Editing. The novel genome editing systems help to introduce stably inherited point modifications into the plant genome, and transgenic region can be easily removed after editing a target gene. This allows creation of nontransgenic plants and improved crop varieties [22, 91-93]. These technologies are faster compared to traditional breeding methods and help to obtain the null segregant lines that have lost the transgene insertion [9497]. Plants with targeted mutations developed by genome editing technology are nearly identical to plants obtained by classical breeding, and their safety must be assessed taking into account the resulting product rather than the process used to create them [98-100]. In this context, ODM-derived products are in many cases indistinguishable from conventionally bred or traditional mutagenesis products; therefore, such products should not be regulated in the same way as the products generated by genetic engineering methods $[7,65]$. Using CRISPR-Cas9 system, it becomes possible to obtain marker-free genetically engineered crops, that is, without marker genes of antibiotic resistance $[6,100]$. Thus, in the case of new varieties with targeted mutations, developed using genome editing systems, the existing operating rules for the regulation of genetically modified plants should not be applied $[92,95,99,100]$. Currently genome editing technologies are being discussed by various advisory and regulatory authorities in the context of GMO legislation. Cultures and plants obtained using genome editing techniques are considered as nongenetically modified $[95,99,101]$. The European Commission is expected to publish a report on regulatory uncertainty of genome editing methods [100, 102, 103].

\section{Multitude of Advantages and Perspectives of GEENs}

Tools of genome editing have a significant impact on basic and applied research in plant biology [24, 43, 44, 73]. The simplified approach to gene/genome editing represents a valuable tool for plant researchers in functional analysis of gene(s) and for breeders in the integration of key genes in the genomes of agriculturally important crops. Genome editing systems have several attractive features including simplicity, efficiency, high specificity, minimal nontarget effects, and amenability to multiplexing and thus are very promising for use in plant breeding [6].

Site-directed mutagenesis of different genes can provide important information about their functions. Simultaneous targeting of multiple genes/loci by applying multiplex strategies can promote research to identify the role of individual genes in the intracellular signaling pathways and aid in the engineering of complex, multigenic agronomic traits in crops. The preferred use of CRISPR-Cas9 system can be exemplified in completely knockout gene function $[6$, 64], microRNA knockdown screening [6], and programmed editing of certain loci by genome editing systems that can provide a functional separation of cis- and trans-regulatory elements/factors with high accuracy [6]. Another prospective application of CRISPR-Cas9 system may be its use in the formation of conditional alleles, providing spatial and temporal control of gene expression to study the function of lethal genes. Use of inducible or tissue-specific promoters for expression of Cas9 and/or sgRNA can be instrumental for gene expression regulation in a specific tissue, in development stage, or in different environmental conditions [6].

CRISPR-Cas system opens up wide possibilities for labeling endogenous genes with fluorescent proteins to visualize their expression in vivo. Using fluorescent labeled dCas9, changes of genome dynamics/chromosome architectural changes during plant development and their response to environmental stimuli can be learned. These technologies can also be used for the selection of the specific cell types that greatly facilitate the study of various functional aspects [6]. Use of dCas9 can provide a new platform for the selection of activation/repression effector domains to specific genomic loci for regulating endogenous gene expression.

In addition, these technologies can be successfully used in the work on epigenome editing via the selection of proteins responsible for histone modification and DNA methylation, which has emerged as a new way of regulating cellular functions in plants [25]. For the purpose of understanding epigenetic regulation, CRISPR-Cas9 system can also be used for the enrichment of chromatin target sites for the identification of proteins attached to enriched chromatin. Likewise, CRISPR-Cas 9 can be used as a tool to identify regulatory proteins binding to specific DNA sequences controlling the expression of genes. 


\section{Conclusion}

Genome editing tools are becoming popular molecular tools of choice for functional genomics as well as crop improvement. Many examples exist currently where these editing systems are being harnessed for unprecedented understanding of plant biology and crop yield improvement through rapid and targeted mutagenesis and associated breeding $[102,104]$. Because of their several attractive features such as simplicity, efficiency, high specificity, and amenability to multiplexing, genome editing technologies described here are revolutionizing the way crop breeding is done and paving the way for the next generation breeding.

\section{Conflicts of Interest}

The authors declare that there are no conflicts of interest regarding the publication of this article.

\section{Acknowledgments}

The authors thank Academy of Sciences of Uzbekistan and Science and Technology Agency of Uzbekistan for Research Grants nos. FA-F5-021 and FA-F5-025.

\section{References}

[1] M. F. Singer, "Introduction and historical background," in Genetic Engineering, J. K. Setlow and A. Hollaender, Eds., vol. 1, pp. 1-13, Plenum, New York, NY, USA, 1979.

[2] A. A. Nemudryi, K. R. Valetdinova, S. P. Medvedev, and S. M. Zakian, "TALEN and CRISPR/Cas genome editing systems: tools of discovery," Acta Naturae, vol. 6, no. 22, pp. 19-40, 2014.

[3] Y.-G. Kim, J. Cha, and S. Chandrasegaran, "Hybrid restriction enzymes: zinc finger fusions to Fok I cleavage domain," Proceedings of the National Academy of Sciences of the United States of America, vol. 93, no. 3, pp. 1156-1160, 1996.

[4] T. Gaj, C. A. Gersbach, and C. F. Barbas III, "ZFN, TALEN, and CRISPR/Cas-based methods for genome engineering," Trends in Biotechnology, vol. 31, no. 7, pp. 397-405, 2013.

[5] D. P. Weeks, M. H. Spalding, and B. Yang, "Use of designer nucleases for targeted gene and genome editing in plants," Plant Biotechnology Journal, vol. 14, no. 2, pp. 483-495, 2016.

[6] V. Kumar and M. Jain, "The CRISPR-Cas system for plant genome editing: Advances and opportunities," Journal of Experimental Botany, vol. 66, no. 1, pp. 47-57, 2015.

[7] N. J. Sauer, J. Mozoruk, R. B. Miller et al., "Oligonucleotidedirected mutagenesis for precision gene editing," Plant Biotechnology Journal, vol. 14, no. 2, pp. 496-502, 2016.

[8] J. F. Li, J. E. Norville, and J. Aach, "Multiplex and homologous recombination-mediated genome editing in Arabidopsis and Nicotiana benthamiana using guide RNA and Cas9," Nature Biotechnolog, vol. 31, no. 8, pp. 688-691, 2013.

[9] M. Jinek, K. Chylinski, I. Fonfara, M. Hauer, J. A. Doudna, and E. Charpentier, "A programmable dual-RNA-guided DNA endonuclease in adaptive bacterial immunity," Science, vol. 337, no. 6096, pp. 816-821, 2012.

[10] B. Chen, J. Hu, R. Almeida et al., "Expanding the CRISPR imaging toolset with Staphylococcus aureus Cas9 for simultaneous imaging of multiple genomic loci," Nucleic Acids Research, vol. 44, no. 8, p. e75, 2016.

[11] S. W. Cho, S. Kim, J. M. Kim, and J.-S. Kim, "Targeted genome engineering in human cells with the Cas9 RNA-guided endonuclease," Nature Biotechnology, vol. 31, no. 3, pp. 230-232, 2013.

[12] A. Noman, M. Aqeel, and S. He, "CRISPR-Cas9: Tool for qualitative and quantitative plant genome editing," Frontiers in Plant Science, vol. 7, no. 2016, article no. 1740, 2016.

[13] Y. Mao, H. Zhang, N. Xu, B. Zhang, F. Gou, and J.-K. Zhu, "Application of the CRISPR-Cas system for efficient genome engineering in plants," Molecular Plant, vol. 6, no. 6, pp. 20082011, 2013.

[14] P. D. Hsu, D. A. Scott, J. A. Weinstein et al., "DNA targeting specificity of RNA-guided Cas9 nucleases," Nature Biotechnol, vol. 31, pp. 827-832, 2013.

[15] K. Osakabe, Y. Osakabe, and S. Toki, "Site-directed mutagenesis in Arabidopsis using custom-designed zinc finger nucleases," Proceedings of the National Academy of Sciences of the United States of America, vol. 107, no. 26, pp. 12034-12039, 2010.

[16] F. Zhang, M. L. Maeder, E. Unger-Wallaced et al., "High frequency targeted mutagenesis in Arabidopsis thaliana using zinc finger nucleases," Proceedings of the National Academy of Sciences of the United States of America, vol. 107, no. 26, pp. 12028-12033, 2010.

[17] J. A. Townsend, D. A. Wright, R. J. Winfrey et al., "Highfrequency modification of plant genes using engineered zincfinger nucleases," Nature, vol. 459, no. 7245, pp. 442-445, 2009.

[18] V. K. Shukla, Y. Doyon, J. C. Miller et al., "Precise genome modification in the crop species Zea mays using zinc-finger nucleases," Nature, vol. 459, pp. 437-441, 2009.

[19] M. Christian, Y. Qi, Y. Zhang, and D. F. Voytas, “Targeted Mutagenesis of Arabidopsis thaliana Using Engineered TAL Effector Nucleases," G3: Genes, Genomes, Genetics, vol. 3, no. 9, pp. 1697-1705, 2013.

[20] H. Zhang, F. Gou, J. Zhang et al., “TALEN-mediated targeted mutagenesis produces a large variety of heritable mutations in rice," Plant Biotechnology Journal, vol. 14, no. 1, pp. 186-194, 2016.

[21] T. Li, B. Liu, M. H. Spalding, D. P. Weeks, and B. Yang, "High-efficiency TALEN-based gene editing produces diseaseresistant rice," Nature Biotechnology, vol. 30, no. 5, pp. 390-392, 2012.

[22] M. M. Mahfouz, L. Li, M. Piatek et al., "Targeted transcriptional repression using a chimeric TALE-SRDX repressor protein," Plant Molecular Biology, vol. 78, no. 3, pp. 311-321, 2012.

[23] J. Gao, G. Wang, S. Ma et al., "CRISPR/Cas9-mediated targeted mutagenesis in Nicotiana tabacum," Plant Molecular Biology, vol. 87, no. 1-2, pp. 99-110, 2015.

[24] L. Cong, F. A. Ran, D. Cox et al., "Multiplex genome engineering using CRISPR/Cas systems," Science, vol. 339, no. 6121, pp. 819823, 2013.

[25] H. Puchta, "Using CRISPR/Cas in three dimensions: towards synthetic plant genomes, transcriptomes and epigenomes," Plant Journal, vol. 87, no. 1, pp. 5-15, 2016.

[26] T. B. Jacobs, P. R. LaFayette, R. J. Schmitz, and W. A. Parrott, "Targeted genome modifications in soybean with CRISPR/Cas9," BMC Biotechnology, pp. 1-10, 2015.

[27] R. Xu, R. Qin, H. Li et al., "Generation of targeted mutant rice using a CRISPR-Cpf1 system," Plant Biotechnology Journal, vol. 14, pp. 1-5, 2016. 
[28] W. Z. Jiang, I. M. Henry, P. G. Lynagh, L. Comai, E. B. Cahoon, and D. P. Weeks, "Significant enhancement of fatty acid composition in seeds of the allohexaploid, Camelina sativa, using CRISPR/Cas9 gene editing," Plant Biotechnology Journal, vol. 15, no. 5, pp. 648-657, 2017.

[29] S. Soyk, N. A. Müller, S. J. Park et al., "Variation in the flowering gene SELF PRUNING 5G promotes day-neutrality and early yield in tomato," Nature Genetics, vol. 49, no. 1, pp. 162-168, 2017.

[30] J. Peng, D. E. Richards, and N. M. Hartley, "Green revolution genes encode mutant gibberellin response modulators," Nature, vol. 400, no. 6741, pp. 256-261, 1999.

[31] Y. Osakabe, T. Watanabe, S. S. Sugano et al., "Optimization of CRISPR/Cas9 genome editing to modify abiotic stress responses in plants," Scientific Reports, vol. 6, Article ID 26685, 2016.

[32] Q. Shan, Y. Wang, and J. Li, "Targeted genome modification of crop plants using a CRISPR-Cas system," Nature Biotechnol, vol. 31, pp. 686-688, 2013.

[33] S. Svitashev, J. K. Young, C. Schwartz, H. Gao, S. C. Falco, and A. M. Cigan, "Targeted mutagenesis, precise gene editing, and sitespecific gene insertion in maize using Cas9 and guide RNA," Plant Physiology, vol. 169, no. 2, pp. 931-945, 2015.

[34] Y. Wang, X. Cheng, and Q. Shan, "Simultaneous editing of three homoeoalleles in hexaploid bread wheat confers heritable resistance to powdery mildew," Nature Biotechnol, vol. 32, pp. 947-952, 2014.

[35] X. Ji, H. Zhang, Y. Zhang, Y. Wang, and C. Gao, "Establishing a CRISPR-Cas-like immune system conferring DNA virus resistance in plants," Nature Plants, vol. 1, article 15144, Article ID 15144, 2015.

[36] Z. Ali, A. Abulfaraj, A. Idris, S. Ali, M. Tashkandi, and M. M. Mahfouz, "CRISPR/Cas9-mediated viral interference in plants," Genome Biology, vol. 16, no. 1, article 238, 2015.

[37] N. J. Baltes, A. W. Hummel, and E. Konecna, "Conferring resistance to geminiviruses with the CRISPR-Cas prokaryotic immune system," Nature Plants, vol. 1, article 15145, 2015.

[38] D. Liu, X. Chen, J. Liu, J. Ye, and Z. Guo, “The rice ERF transcription factor OsERF922 negatively regulates resistance to Magnaporthe oryzae and salt tolerance," Journal of Experimental Botany, vol. 63, no. 10, pp. 3899-3912, 2012.

[39] F. Wang, C. Wang, P. Liu et al., "Enhanced rice blast resistance by CRISPR/ Cas9-Targeted mutagenesis of the ERF transcription factor gene OsERF922," PLoS ONE, vol. 11, no. 4, Article ID e0154027, 2016.

[40] J. Chandrasekaran, M. Brumin, D. Wolf et al., "Development of broad virus resistance in non-transgenic cucumber using CRISPR/Cas9 technology," Molecular Plant Pathology, vol. 17, no. 7, pp. 1140-1153, 2016.

[41] F. Zhang, Y. Wen, and X. Guo, "CRISPR/Cas9 for genome editing: Progress, implications and challenges," Human Molecular Genetics, vol. 23, no. 1, pp. R40-R46, 2014.

[42] J. F. Petolino and J. P. Davies, "Designed transcriptional regulators for trait development," Plant Science, vol. 201-202, no. 1, pp. 128-136, 2013.

[43] H. Wang, H. Yang, C. S. Shivalila et al., "One-step generation of mice carrying mutations in multiple genes by CRISPR/Casmediated genome engineering," Cell, vol. 153, no. 4, pp. 910-918, 2013.

[44] L. Lowder, A. Malzahn, and Y. Qi, "Rapid evolution of manifold CRISPR systems for plant genome editing," Frontiers in Plant Science, vol. 7, no. 2016, article no. 1683, 2016.
[45] D. G. Knorre and V. V. Vlasov, "Reactive derivatives of nucleic acids and their components as affinity reagents," Russian Chemical Reviews, vol. 54, no. 9, pp. 836-851, 1985.

[46] N. J. Palpant and D. Dudzinski, "Zinc finger nucleases: Looking toward translation," Gene Therapy, vol. 20, no. 2, pp. 121-127, 2013.

[47] R. Jankele and P. Svoboda, "TAL effectors: Tools for DNATargeting," Briefings in Functional Genomics, vol. 13, no. 5, pp. 409-419, 2014.

[48] C. O. Pabo, E. Peisach, and R. A. Grant, "Design and selection of novel Cys2His2 zinc finger proteins," Annual Review of Biochemistry, vol. 70, pp. 313-340, 2001.

[49] T. Cathomen and J. Keith Joung, "Zinc-finger nucleases: the next generation emerges," Molecular Therapy, vol. 16, no. 7, pp. 1200-1207, 2008.

[50] J. F. Petolino, "Genome editing in plants via designed zinc finger nucleases," In Vitro Cellular and Developmental Biology - Plant, vol. 51, no. 1, 2015.

[51] N. P. Pavletich and C. O. Pabo, "Zinc finger-DNA recognition: crystal structure of a Zif268-DNA complex at 2.1 Å," Science, vol. 252, no. 5007, pp. 809-817, 1991.

[52] W. M. Ainley, L. Sastry-Dent, M. E. Welter et al., "Trait stacking via targeted genome editing," Plant Biotechnology Journal, vol. 11, no. 9, pp. 1126-1134, 2013.

[53] J. F. Petolino, A. Worden, K. Curlee et al., "Zinc finger nucleasemediated transgene deletion," Plant Molecular Biology, vol. 73, no. 6, pp. 617-628, 2010.

[54] S. Schornack, A. Meyer, P. Römer, T. Jordan, and T. Lahaye, "Gene-for-gene-mediated recognition of nuclear-targeted AvrBs3-like bacterial effector proteins," Journal of Plant Physiology, vol. 163, no. 3, pp. 256-272, 2006.

[55] P. Römer, S. Hahn, T. Jordan, T. Strauß, U. Bonas, and T. Lahaye, "Plant pathogen recognition mediated by promoter activation of the pepper Bs3 resistance gene," Science, vol. 318, no. 5850, pp. 645-648, 2007.

[56] J. Boch, H. Scholze, S. Schornack et al., "Breaking the code of DNA binding specificity of TAL-type III effectors," Science, vol. 326, no. 5959, pp. 1509-1512, 2009.

[57] B. M. Lamb, A. C. Mercer, and C. F. Barbas III, "Directed evolution of the TALE N-terminal domain for recognition of all 50 bases," Nucleic Acids Research, vol. 41, no. 21, pp. 9779-9785, 2013.

[58] M. Christian, T. Cermak, E. L. Doyle et al., "Targeting DNA double-strand breaks with TAL effector nucleases," Genetics, vol. 186, no. 2, pp. 757-761, 2010.

[59] L. Cong, R. H. Zhou, Y.-C. Kuo, M. Cunniff, and F. Zhang, "Comprehensive interrogation of natural TALE DNA-binding modules and transcriptional repressor domains," Nature Communications, vol. 3, article 968, 2012.

[60] M. L. Christian, Z. L. Demorest, C. G. Starker et al., “Targeting G with TAL Effectors: A Comparison of Activities of TALENs Constructed with NN and NK Repeat Variable Di-Residues," PLoS ONE, vol. 7, no. 9, Article ID e45383, 2012.

[61] J. Streubel, C. Blücher, A. Landgraf, and J. Boch, "TAL effector RVD specificities and efficiencies," Nature Biotechnology, vol. 30, no. 7, pp. 593-595, 2012.

[62] A. N.-S. Mak, P. Bradley, R. A. Cernadas, A. J. Bogdanove, and B. L. Stoddard, "The crystal structure of TAL effector PthXol bound to its DNA target," Science, vol. 335, no. 6069, pp. 716719, 2012. 
[63] J. Xiong, J. Ding, and Y. Li, "Genome-editing technologies and their potential application in horticultural crop breeding," Horticulture Research, vol. 2, article 15019, 2015.

[64] I. Y. Abdurakhmonov, "Genomics Era for Plants and Crop Species-Advances Made and Needed Tasks Ahead," in Plant Genomics, I. Abdurakhmonov, Ed., InTech, Croatia, Balkans, 2016.

[65] CropLife International, "Oligonucleotide-Directed Mutagenesis (ODM)," LJournal, 2017.

[66] R. Barrangou, C. Fremaux, and H. Deveau, "CRISPR provides acquired resistance against viruses in prokaryotes," Science, vol. 315, no. 5819, pp. 1709-1712, 2007.

[67] E. Deltcheva, K. Chylinski, C. M. Sharma et al., "CRISPR RNA maturation by trans-encoded small RNA and host factor RNase III," Nature, vol. 471, no. 7340, pp. 602-607, 2011.

[68] D. H. Haft, J. Selengut, E. F. Mongodin, and K. E. Nelson, "A guild of 45 CRISPR-associated (Cas) protein families and multiple CRISPR/cas subtypes exist in prokaryotic genomes," PLoS Computational Biology, vol. 1, article e60, no. 6, pp. 04740483, 2005.

[69] A. F. Gilles and M. Averof, "Functional genetics for all: Engineered nucleases, CRISPR and the gene editing revolution," EvoDevo, vol. 5, no. 1, article no. 43, 2014.

[70] J. A. Doudna and E. Charpentier, "The new frontier of genome engineering with CRISPR-Cas9,” Science, vol. 346, no. 6213, 2014.

[71] D. B. Graham and D. E. Root, "Resources for the design of CRISPR gene editing experiments," Genome Biology, vol. 16, no. 1, article no. 260, 2015.

[72] L. C. Perkin, S. L. Adrianos, and B. Oppert, "Gene disruption technologies have the potential to transform stored product insect pest control," Insects, vol. 7, no. 3, article no. 46, 2016.

[73] P. Perez-Pinera, D. G. Ousterout, and C. A. Gersbach, "Advances in targeted genome editing," Current Opinion in Chemical Biology, vol. 16, no. 3-4, pp. 268-277, 2012.

[74] L. Chen, L. Tang, H. Xiang et al., "Advances in genome editing technology and its promising application in evolutionary and ecological studies," GigaScience, vol. 3, no. 1, article no. 24, 2014.

[75] C. Kissoudis, C. van de Wiel, R. G. F. Visser, and G. van der Linden, "Enhancing crop resilience to combined abiotic and biotic stress through the dissection of physiological and molecular crosstalk," Frontiers in Plant Science, vol. 5, no. MAY, article no. 207, 2014.

[76] L. Liu and X.-D. Fan, "CRISPR-Cas system: A powerful tool for genome engineering," Plant Molecular Biology, vol. 85, no. 3, pp. 209-218, 2014.

[77] M. Jain, "Function genomics of abiotic stress tolerance in plants: A CRISPR approach," Frontiers in Plant Science, vol. 6, no. MAY, article no. 375, pp. 1-4, 2015.

[78] G. Andolfo, P. Iovieno, L. Frusciante, and M. R. Ercolano, "Genome-editing technologies for enhancing plant disease resistance," Frontiers in Plant Science, vol. 7, no. 2016, article no. $1813,2016$.

[79] S. Khatodia, K. Bhatotia, N. Passricha, S. M. P. Khurana, and N. Tuteja, "The CRISPR/Cas genome-editing tool: Application in improvement of crops," Frontiers in Plant Science, vol. 7, no. 2016, article no. 506, 2016.

[80] R. C. Nongpiur, S. L. Singla-Pareek, and A. Pareek, "Genomics Approaches for Improving Salinity Stress Tolerance in Crop Plants," Current Genomics, vol. 17, no. 4, pp. 343-357, 2016.
[81] V. Shukla, M. Gupta, F. Urnov, D. Guschin, M. Jan, and P. Bundock, "Targeted modifcation of malate dehydrogenase, 2013," WO Patent Publication Number: WO 2013166315 A1.

[82] C. A. Hollender and C. Dardick, "Molecular basis of angiosperm tree architecture," New Phytologist, vol. 206, no. 2, pp. 541-556, 2015.

[83] Y. Fang and B. M. Tyler, "Efficient disruption and replacement of an effector gene in the oomycete Phytophthora sojae using CRISPR/Cas9," Molecular Plant Pathology, vol. 17, no. 1, pp. 127139, 2016.

[84] G. E. Hastings and P. G. Wolf, "The Therapeutic Use of Albumin," Archives of Family Medicine, vol. 1, no. 2, pp. 281-287, 1992.

[85] Y. He, T. Ning, T. Xie et al., "Large-scale production of functional human serum albumin from transgenic rice seeds," Proceedings of the National Academy of Sciences of the United States of America, vol. 108, no. 47, pp. 19078-19083, 2011.

[86] M. Bosch and S. P. Hazen, "Lignocellulosic feedstocks: Research progress and challenges in optimizing biomass quality and yield," Frontiers in Plant Science, vol. 4, article no. 474, 2013.

[87] C. M. Lee, T. J. Cradick, E. J. Fine, and G. Bao, "Nuclease target site selection for maximizing on-target activity and minimizing off-target effects in genome editing," Molecular Therapy, vol. 24, no. 3, pp. 475-487, 2016.

[88] Q. C. Cai, J. Miller, F. Urnov et al., "Optimized non-canonical zinc finger proteins,” US Patent Number: 9,187,758. Publication date: Nov 17, 2015.

[89] A. Lombardo, D. Cesana, P. Genovese et al., "Site-specific integration and tailoring of cassette design for sustainable gene transfer," Nature Methods, vol. 8, no. 10, pp. 861-869, 2011.

[90] T. Koo, J. Lee, and J. Kim, "Measuring and reducing off-target activities of programmable nucleases including CRISPR-Cas9," Molecules and Cells, vol. 38, no. 6, pp. 475-481, 2015.

[91] Y. Gao and Y. Zhao, "Specific and heritable gene editing in Arabidopsis," Proceedings of the National Academy of Sciences of the United States of America, vol. 111, no. 12, pp. 4357-4358, 2014.

[92] C. Nagamangala Kanchiswamy, D. J. Sargent, R. Velasco, M. E. Maffei, and M. Malnoy, "Looking forward to genetically edited fruit crops," Trends in Biotechnology, vol. 33, no. 2, pp. 62-64, 2015.

[93] R.-F. Xu, H. Li, R.-Y. Qin et al., "Generation of inheritable and "transgene clean" targeted genome-modified rice in later generations using the CRISPR/Cas9 system," Scientific Reports, vol. 5, Article ID 11491, 2015.

[94] N. Podevin, Y. Devos, H. V. Davies, and K. M. Nielsen, "Transgenic or not? No simple answer! New biotechnologybased plant breeding techniques and the regulatory landscape," EMBO Reports, vol. 13, no. 12, pp. 1057-1061, 2012.

[95] M. Araki and T. Ishii, "Towards social acceptance of plant breeding by genome editing," Trends in Plant Science, vol. 20, no. 3, pp. 145-149, 2015.

[96] J. G. Schaart, C. C. M. van de Wiel, L. A. P. Lotz, and M. J. M. Smulders, "Opportunities for Products of New Plant Breeding Techniques," Trends in Plant Science, vol. 21, no. 5, pp. 438-449, 2016.

[97] J. W. Woo, J. Kim, S. I. Kwon et al., "DNA-free genome editing in plants with preassembled CRISPR-Cas9 ribonucleoproteins," Nature Biotechnology, vol. 33, no. 11, pp. 1162-1164, 2015.

[98] F. Hartung and J. Schiemann, "Precise plant breeding using new genome editing techniques: Opportunities, safety and 
regulation in the EU," Plant Journal, vol. 78, no. 5, pp. 742-752, 2014.

[99] D. F. Voytas and C. Gao, "Precision genome engineering and agriculture: opportunities and regulatory challenges," PLoS biology, vol. 12, no. 6, p. e1001877, 2014.

[100] H. D. Jones, "Regulatory uncertainty over genome editing," Nature Plants, vol. 1, Article ID 14011, 2015.

[101] M. Lusser, C. Parisi, D. Plan, and E. Rodríguez-Cerezo, "Deployment of new biotechnologies in plant breeding," Nature Biotechnology, vol. 30, no. 3, pp. 231-239, 2012.

[102] K. Belhaj, A. Chaparro-Garcia, S. Kamoun, N. J. Patron, and V. Nekrasov, "Editing plant genomes with CRISPR/Cas9," Current Opinion in Biotechnology, vol. 32, pp. 76-84, 2015.

[103] J. D. Wolt, K. Wang, and B. Yang, "The regulatory status of genome-edited crops," Plant Biotechnology Journal, vol. 14, no. 2, pp. 510-518, 2016.

[104] S. Huang, D. Weigel, R. N. Beachy, and J. Li, "A proposed regulatory framework for genome-edited crops," Nature Genetics, vol. 48, no. 2, pp. 109-111, 2016. 


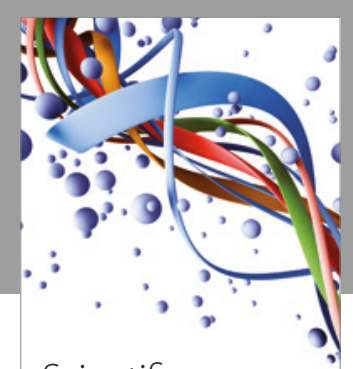

Scientifica
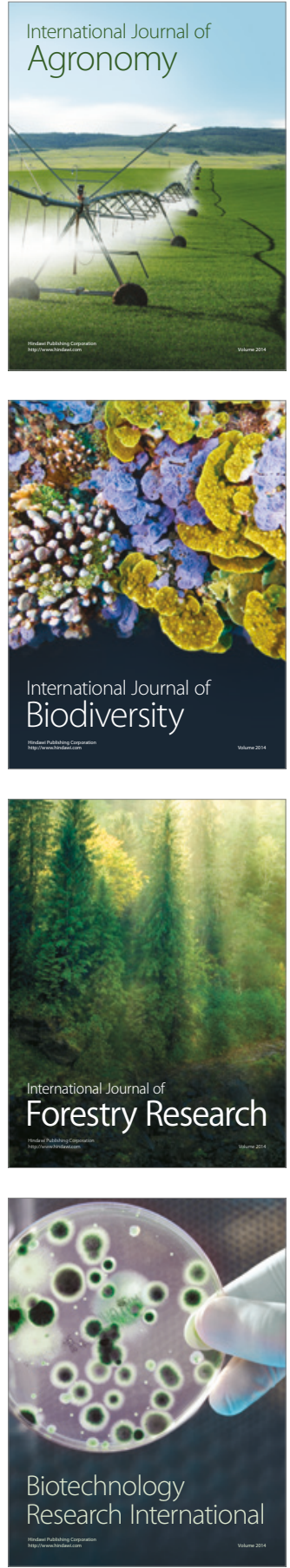
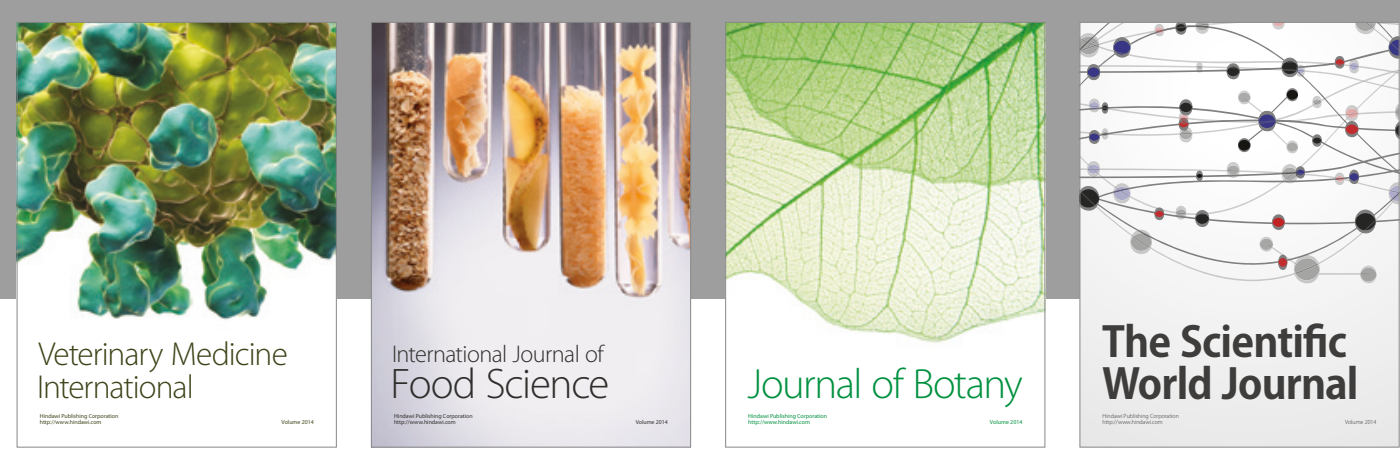

The Scientific

\section{World Journal}

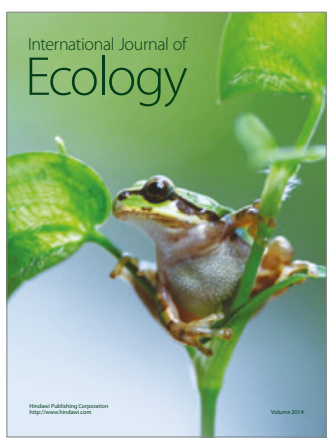

\section{Hindawi}

Submit your manuscripts at

https://www.hindawi.com
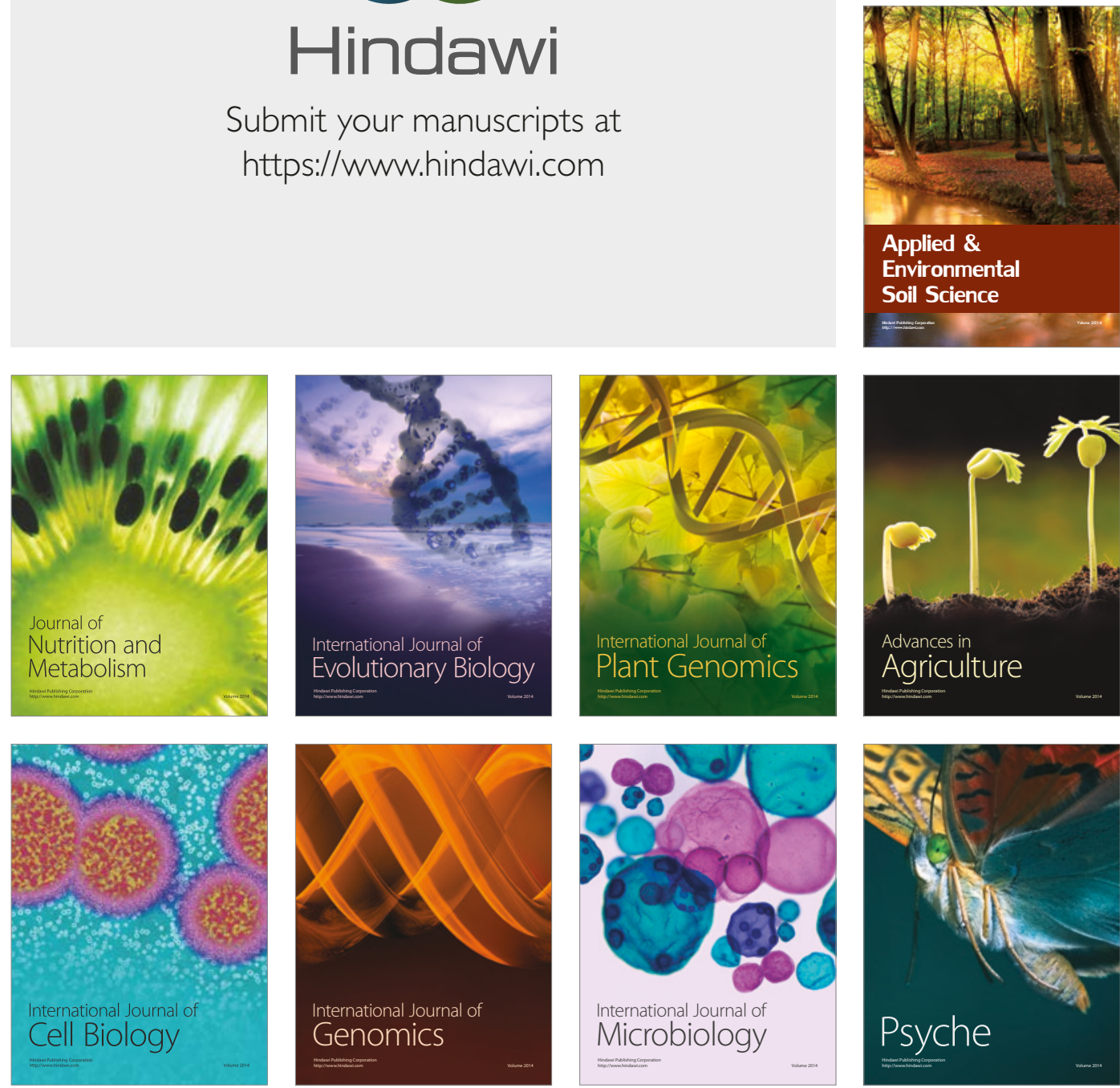

hternational Journal of Microbiology
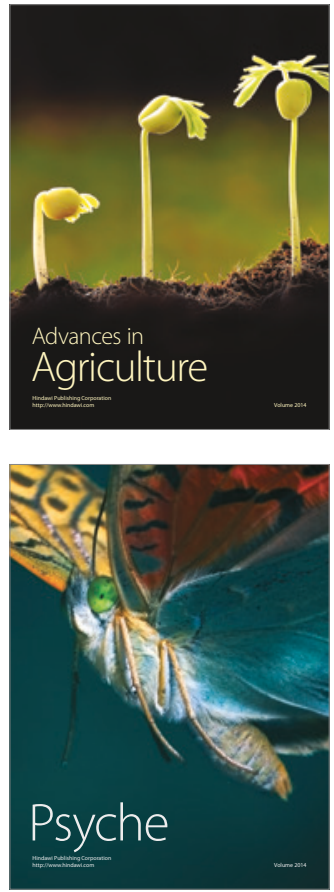